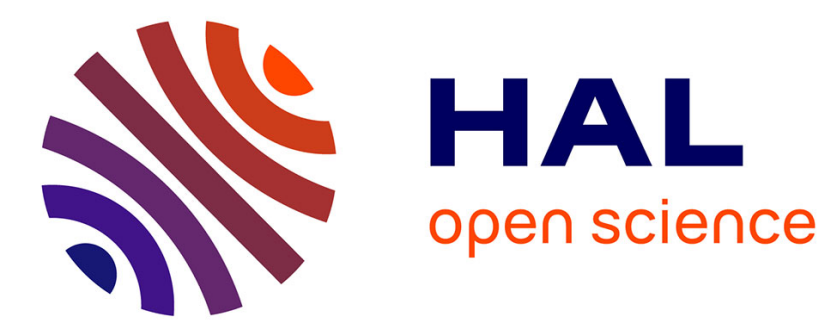

\title{
Low complexity iterative receiver for linear precoded MIMO systems
}

Pierre-Jean Bouvet, Maryline Hélard, Vincent Le Nir

\section{To cite this version:}

Pierre-Jean Bouvet, Maryline Hélard, Vincent Le Nir. Low complexity iterative receiver for linear precoded MIMO systems. 2004, 5 p. hal-00005809

\section{HAL Id: hal-00005809 \\ https://hal.science/hal-00005809}

Submitted on 4 Jul 2005

HAL is a multi-disciplinary open access archive for the deposit and dissemination of scientific research documents, whether they are published or not. The documents may come from teaching and research institutions in France or abroad, or from public or private research centers.
L'archive ouverte pluridisciplinaire HAL, est destinée au dépôt et à la diffusion de documents scientifiques de niveau recherche, publiés ou non, émanant des établissements d'enseignement et de recherche français ou étrangers, des laboratoires publics ou privés. 


\title{
Low complexity iterative receiver for linear precoded MIMO systems
}

\author{
Pierre-Jean Bouvet, Maryline Hélard, Member, IEEE, Vincent Le Nir \\ France Telecom R\&D \\ 4 rue du Clos Courtel 35512 Césson-Sévigné France \\ Email: pierrejean.bouvet@rd.francetelecom.com
}

\begin{abstract}
In a Multiple Input Multiple Output (MIMO) context, we present, in this paper, a Space-Time Bit Interleaved Coded Modulation (STBICM) system combined with a spacetime spreader called linear precoding. We propose a practical low-complexity receiver structure performing iteratively space-time detection and channel decoding. The novelty lies in the linearity of the space-time detector which consists of an MMSE MIMO PSIC equalizer and a linear deprecoder. Spectral efficiency and diversity can easily be tuned while keeping feasible receiver. In the case of independent flat Rayleigh fading, simulation results show that such system exploits both spatial diversity and capacity provided by the multi antenna system.
\end{abstract}

\section{INTRODUCTION}

Schemes deploying multiple transmit and multiple receive antennas have received a lot of attention since it was shown, with the pioneering work of Foschini and Gans, that the capacity of a such wireless link can be linearly increased [1]. In [2], a first system based on Layered Space-Time (LST) architecture at the receiver, is designed in order to exploit such capacity. Interesting improvement can be found in [3] where channel coding is concatenated with LST architecture and where an efficient receiver based on iterative decoding is proposed.

In other hand, the use of diversity techniques is an efficient way to combat detrimental effects of fading channels. Transmit and receive diversity, provided by using multiple transmit and/or receive antennas is a very promising candidate for this. An efficient method to exploit such a diversity, known as space-time coding, was proposed in [4]. A trellis based approach was first studied before the block one i.e Space Time Block Coding (STBC) emerged [5]. Space time coding schemes provide full diversity but do not improve data rate. In [6], A. Tonello presents another approach, called SpaceTime Bit Interleaving Coded Modulation (STBICM). Proposed principle is based on a multi-transmit antennas extension of the Bit-Interleaved Coded Modulation (BICM) concept [7]. At the transmitter, a channel encoder, a bit interleaver, a binary M-ary encoder and a space-time mapper are serially concatenated. The decoding scheme uses an iterative process where extrinsic information are exchanged between a soft-in soft-out MIMO demapper and a soft-in soft-out channel decoder. The main interest of such system is to increase both spectral efficiency and transmission quality. However the optimal space-time demapper leads to a complex exhaustive algorithm based on maximum a priori strategy.

In parallel, linear precoding was demonstrated to efficiently exploit time or space-time diversity [8][9]. In [10] linear precoding is combining with STBICM requiring a Maximum Likelihood (ML) decoder which complexity increases exponentially with the number of receive/transmit antennas, the modulation order and the precoding matrix size. In [11], a particular precoding matrix is combined with orthogonal STBC leading to a low complexity linear decoder and a very efficient exploitation of diversity.

In this paper, we propose to use precoding matrix presented in [11] but associated with STBICM, instead of STBC, in order to improve space-time diversity by increasing the size of precoding matrix. The linear precoding operates as a spacetime spreader on transmitted symbols. At the receiver, we propose an iterative linear receiver offering an interesting trade-off between BER performance and complexity.

We first describe the linear precoding in Section II. The transmitter and the channel model are introduced in Section III. Section IV is devoted to the proposed receiver and finally, Section V provides simulations results.

\section{LINEAR PRECODING}

In this section, we present the linear precoding according to the construction described in [11] which allows an efficient exploitation of the spatial diversity at a linearly cost.

Let $\underline{\mathbf{x}}=\left[x_{1} x_{2} \ldots x_{L}\right]^{T}$, a vector of $L$ complex symbols to be transmitted. These symbols are linear precoded by applying the following complex Hadamard construction matrix based on $\mathrm{SU}(2)$ group matrix of size $L \times L$ :

$$
\boldsymbol{\Theta}_{L}^{H a d}=\sqrt{\frac{2}{L}}\left[\begin{array}{cc}
\boldsymbol{\Theta}_{L / 2} & \boldsymbol{\Theta}_{L / 2} \\
\boldsymbol{\Theta}_{L / 2} & -\boldsymbol{\Theta}_{L / 2}
\end{array}\right]
$$

with $L=2^{n}, \mathrm{n} \in \mathbf{N}^{*}, \mathrm{n} \geq 2$ and $\boldsymbol{\Theta}_{\mathbf{2}}$ belonging to the Special Unitary group $\mathrm{SU}(2)$, therefore $\operatorname{det}\left(\boldsymbol{\Theta}_{\mathbf{2}}\right)=1$ and $\Theta_{2}^{-1}=\Theta_{2}^{H}$. 
The linear precoded symbols vector $\mathbf{s}$ is given by:

$$
\underline{\mathbf{s}}=\boldsymbol{\Theta}_{L} \cdot \underline{\mathbf{x}}
$$

with $\underline{\mathbf{s}}=\left[\begin{array}{llll}s_{1} & s_{2} & \ldots & s_{L}\end{array}\right]^{T}$ are the spread symbols.

At the receiver side, estimates of precoded symbols $\underline{\hat{\mathbf{s}}}$ are simply deprecoded by applying the transconjugate precoding matrix:

$$
\underline{\hat{\mathbf{x}}}=\mathbf{\Theta}_{L}^{H} \cdot \underline{\hat{\mathbf{s}}}
$$

where $(.)^{H}$ is the complex transconjugate operand. If $\underline{\hat{\mathbf{s}}}$ is not corrupted by important interference terms, a linear deprecoding was demonstrated to be very efficient [11]. Moreover, we can notice that no channel knowledge is required as well at the precoder as at the deprecoder.

\section{System Model AND NOTATION}

We apply linear precoding to STBICM in order to efficiently exploit both space-time diversity and MIMO capacity.

\section{A. Transmitter}

At the transmitter the information bit stream $d$ is first convolutionally encoded and then bit-interleaved $\left(\Pi_{b}\right)$ to produce the bit stream $c$. The interleaved stream is mapped into complex symbols $x$ belonging to a multi-phase/level constellation $\chi \in \mathbb{C}^{M}$ where $M$ is the modulation order. Each block of $L$ symbols are linearly precoded to a block of precoded symbols $\underline{\mathbf{s}}$ owing to matrix $\Theta_{L}$ introduced in Section II. The latest stream is finally serial to parallel converted into $N_{t}$ streams and simultaneously transmitted each from a different antenna. The spectral efficiency of such a scheme is $\eta=R_{c} \cdot N_{t} \cdot \log _{2} M$ bits $/ \mathrm{s} / \mathrm{Hz}$ with $R_{c}$ convolutional encoder rate. Figure 1 describes the transmitter scheme. We call this system Linear Precoded Space-Time Bit Interleaved Coded Modulation (LP-STBICM)

Although $L$ can be fixed independently of $N_{t}$ or $N_{r}$, in order to simplify the notation, we assume that $L=\alpha N_{t}$ with $\alpha \in \mathbb{N}$ and $N_{t} \geq 1$. Thus, the vector $\underline{\mathbf{s}}$ can be decomposed in the following way: $\underline{\mathbf{s}}=\left[\begin{array}{llll}\mathbf{s}(1) & \mathbf{s}(2) & \ldots & \mathbf{s}\left(L / N_{t}\right)\end{array}\right]^{T}$ where $\mathbf{s}(k)=\left[\begin{array}{llll}s_{1+k} & s_{2+k} & \ldots & s_{N_{t}+k}\end{array}\right]^{T}$ represents the transmitted symbols at time $k$ from all the transmitting antennas.

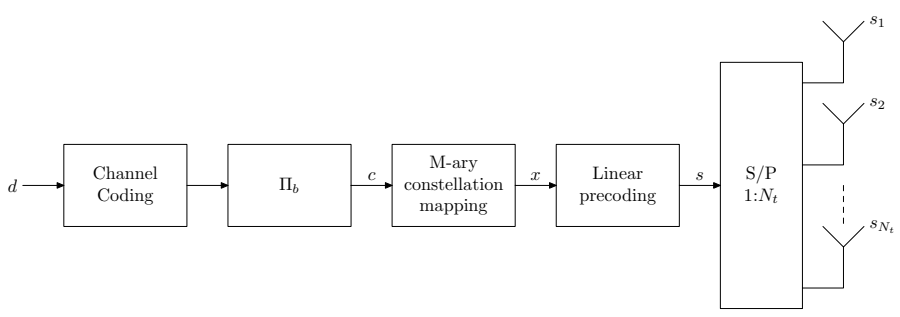

Fig. 1. LP-STBICM Emission

\section{B. MIMO channel}

The MIMO channel we consider connects $N_{t} \geq 2$ transmit antennas with $N_{r} \geq 2$ receive antennas. At time $k$, the channel output is the superposition of the $N_{t}$ transmitted symbols weighted by the channel response. We assume frequency nonselective sub-channels. The attenuation between the transmit antenna $i$ and the receive antenna $j$ is modeled by a complex coefficient $h_{i j}(k)$. All these coefficients are the entries of an $N_{r} \times N_{t}$ channel matrix $\mathbf{H}(k)=\left(h_{i j}(k)\right)$. These coefficients are modelled by independent complex zero-mean Gaussian random variables of unit variance. At each of the receive antennas, the signal is corrupted by complex-valued additive white Gaussian noise (AWGN) of variance $\sigma_{n}^{2}$. At time $k$, all these noise samples are collected in vector $\mathbf{n}(k) \in \mathbb{C}^{N_{r} \times 1}$. The transmit energy is equally distributed over the $N_{t}$ antennas. We assume the channel coefficients and the noise variance are unknown at the transmitter but perfectly known at the receiver.

Let $\mathbf{r}(k) \in \mathbb{C}^{N r \times 1}$ the receive vector at time $k$. We can write:

$$
\mathbf{r}(k)=\mathbf{H}(k) \mathbf{s}(k)+\mathbf{n}(k)
$$

If we note $\underline{\mathbf{H}}=\operatorname{diag}\left\{\mathbf{H}(1), \mathbf{H}(2), \ldots, \mathbf{H}\left(\frac{L}{N_{t}}\right)\right\}$ with $\underline{\mathbf{H}} \in$ $\mathbb{C}^{\frac{N_{r} L}{N_{t}} \times L}$, we have the following equation:

$$
\underline{\mathbf{r}}=\underline{\mathbf{H}} \cdot \underline{\mathbf{s}}+\underline{\mathbf{n}}
$$

where $\underline{\mathbf{r}}=\left[\begin{array}{llll}\mathbf{r}(1) & \mathbf{r}(2) & \ldots & \mathbf{r}\left(\frac{L}{N_{t}}\right)\end{array}\right]^{T}, \underline{\mathbf{r}} \in \mathbb{C}^{\frac{N_{r} L}{N_{t}} \times 1}$ and $\underline{\mathbf{n}}=\left[\begin{array}{llll}\mathbf{n}(1) & \mathbf{n}(2) & \ldots & \mathbf{n}\left(\frac{L}{N_{t}}\right)\end{array}\right]^{T}, \underline{\mathbf{n}} \in \mathbb{C}^{\frac{N_{r} L}{N_{t}} \times 1}$.

\section{ITERATIVE JOINT EQUALIZATION, DEPRECODING AND CHANNEL DECODING}

Optimal signal decoding which would consist of an optimal decoding of a trellis including the effect of channel coding, precoding, interleaving and space-time channel is extremely complex and could not be reasonably implemented. In [10], a sub-optimum detection scheme based on iterative "turbo" detection principle [12] is used. Iterative joint detection and decoding is performed owing to the exchange of soft information between an A Posteriori Probability (APP) detector for both MIMO channel and precoding and a convolutional decoder. However the detector has a complexity growing with the MIMO architecture size, the modulation order and the precoding size.

In this paper, we propose a very low complexity iterative algorithm comprising mainly linear elements inspired from filterbased turbo-equalization [13]. Two main decoding stages, a MIMO demapper and a Soft-In Soft-Out (SISO) channel decoder, exchange the information learned from one stage to another iteratively until the receiver converges. Each stages are separated by interleaver and deinterleaver in order to decorrelate the outputs before feeding them to the next decoding stage. 


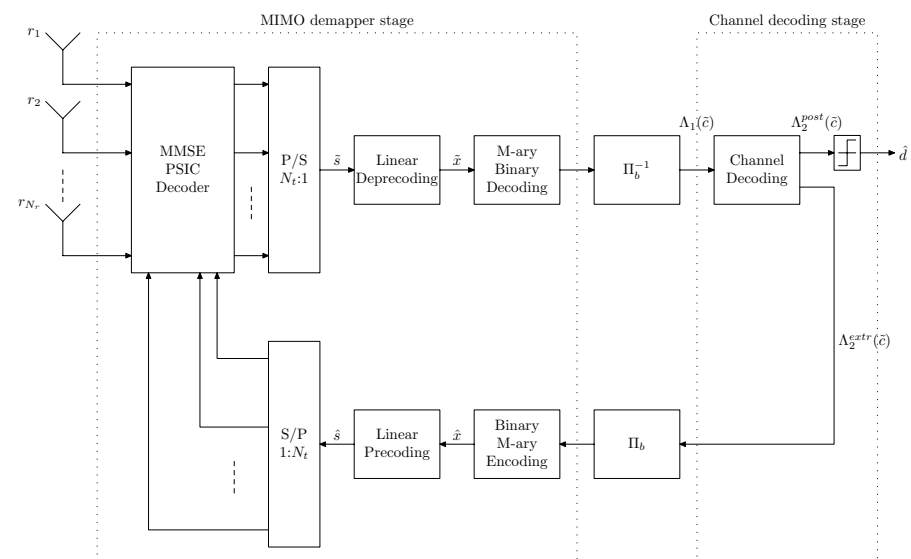

Fig. 2. LP-STBICM Receiver

\section{A. Description of the iterative decoding scheme}

The proposed receiver is depicted in Figure 2. The MIMO demapper stage first consists of a Parallel Soft Interference Canceller (PSIC) equalizer, optimized under the Minimum Mean Square Error (MMSE) criterion, that generates soft estimates $\underline{\tilde{\mathbf{s}}}$ of transmitted signal $\underline{\mathbf{s}}$. After multiplexing, these equalized symbols are deprecoded to produce M-ary data symbol estimates $\underline{\tilde{\tilde{x}}}$. The demapper produces Logarithm Likelihood Ratio (LLR) of coded bits $\Lambda_{1}(\tilde{c})$. These LLRs are fed via a desinterleaving module $\left(\Pi_{b}\right)$ to the channel decoding stage. The channel decoder produces on the one hand a posteriori LLRs on bits $\Lambda_{2}^{\text {post }}(\hat{d})$ that are used to estimate transmitted binary data $\underline{\hat{\mathbf{d}}}$. On the other hand, the channel decoder computes extrinsic LLRs using the Berrou-Adde algorithm [14]:

$$
\Lambda_{2}^{e x t r}(\hat{c})=\ln \frac{\operatorname{Pr}\left\{c=1 / \Lambda_{1}(\tilde{c})\right\}}{\operatorname{Pr}\left\{c=0 / \Lambda_{1}(\tilde{c})\right\}}
$$

These extrinsic information are again interleaved $\left(\Pi_{b}\right)$ and then soft converted to M-ary estimated symbols $\underline{\hat{x}}$ owing to the classical relationship:

$$
\hat{x}=\tanh \left(\frac{\Lambda_{2}^{\text {post }}(\hat{c})}{2}\right)
$$

Finally, estimated symbols are precoded, serial to parallel converted and send to the MMSE MIMO PSIC equalizer as new estimates of transmitted symbols vector $\underline{\hat{\hat{s}}}$

\section{B. MMSE MIMO PSIC equalizer}

At sample time $k$, received signal $\mathbf{r}(k)$ is corrupted by Co Antenna Interferences (CAI) represented by off diagonal terms of $\mathbf{H}$. In order to remove the CAI, we use a Parallel Soft Interference Canceller (PSIC) optimized under the minimum mean square error (MMSE) criterion inspired from [13]. In the following we omit the sampling index $(k)$ to simplify the notations.

At the first iteration because no prior information on transmitted symbols is available, a classical MMSE space linear equalizer is used, leading to the first equalized vector $\mathbf{s}^{(1)} \in$ $\mathbb{C}^{N_{t} \times 1}[15]$

$$
\tilde{\mathbf{s}}^{(1)}=\left(\mathbf{H}^{H} \mathbf{H}+\sigma_{n}^{2} \mathbf{I}\right)^{-1} \mathbf{H}^{H} \mathbf{r}
$$

where $\mathbf{I}$ is the $N_{t} \times N_{t}$ identity matrix.

For next iterations, a soft MMSE interference canceller is performed using symbol estimates provided by previous iterations. The expression of the equalized symbols vector obtained at the $p$-th iteration is the following:

$$
\tilde{\mathbf{s}}^{(p)}=\left(\mathbf{D}+\sigma_{n}^{2} \mathbf{I}\right)^{-1}\left(\mathbf{H}^{H} \mathbf{r}-\mathbf{J} \hat{\mathbf{s}}^{(p-1)}\right)
$$

$\hat{\mathbf{s}}^{(p-1)} \in \mathbb{C}^{N_{t} \times 1}$ is the improved estimate vector of transmitted symbols from iteration $p-1$ whereas $\mathbf{D}$ and $\mathbf{J}$ are $N_{t} \times N_{t}$ complex matrix containing respectively the diagonal elements and the off-diagonal elements of $\mathbf{H}^{H} \mathbf{H}$ :

$$
\begin{gathered}
\mathbf{D}=\operatorname{Diag}\left(\mathbf{H}^{H} \mathbf{H}\right) \quad \text { and } \quad \mathbf{J}=\mathbf{H}^{H} \mathbf{H}-\mathbf{D} \\
\text { V. Simulation Results }
\end{gathered}
$$

For our simulations, we consider a $2 \times 2$ and a $4 \times 4$ MIMO architectures. We use an half rate convolutional encoder with generator $(133,177)_{o}$ and a QPSK constellation. Spectral efficiencies of $2 \mathrm{bits} / \mathrm{s} / \mathrm{Hz}$ and $4 \mathrm{bits} / \mathrm{s} / \mathrm{Hz}$ are respectively achieved. A precoder with size $L=64$ is used. The bit interleaver size is 10000 bits. Simulations provide BER performance of proposed LP-STBICM receiver for both MIMO architectures. In order to highlight the linear precoding performance gain, we carry out performance of an STBICM receiver which is obtained by removing the precoder and the deprecoder in the iterative loop.

For a $4 \times 4$ architecture, Figure 3 shows the convergence of the iterative receiver at a signal to noise ratio (SNR) equal to $4 \mathrm{~dB}$. Asymptotic curves for both STBICM and LPSTBICM systems are plotted as well. These curves, called lower bound, are performed by an ideal receiver that would perfectly remove the CAI. At the beginning of the process, the remaining CAI after PSIC decoding is such as precoding has only little effect: LP-STBICM and STBICM curves are similar. However, by increasing the number of iterations, the $\mathrm{CAI}$ is progressively removed and precoding is more and more efficient. We consider that convergence is reached at the end of 5 iterations.

BER performance at the $5^{t h}$ iteration for $2 \times 2$ and $4 \times 4$ MIMO channel are respectively carried out in Figure 4 and Figure 5. At low SNR, the PSIC equalizer doesn't succeed in removing all the CAI, this explains the lost in $\mathrm{dB}$ comparatively to the asymptotic curve. This phenomena is emphasized for $4 \times 4$ 


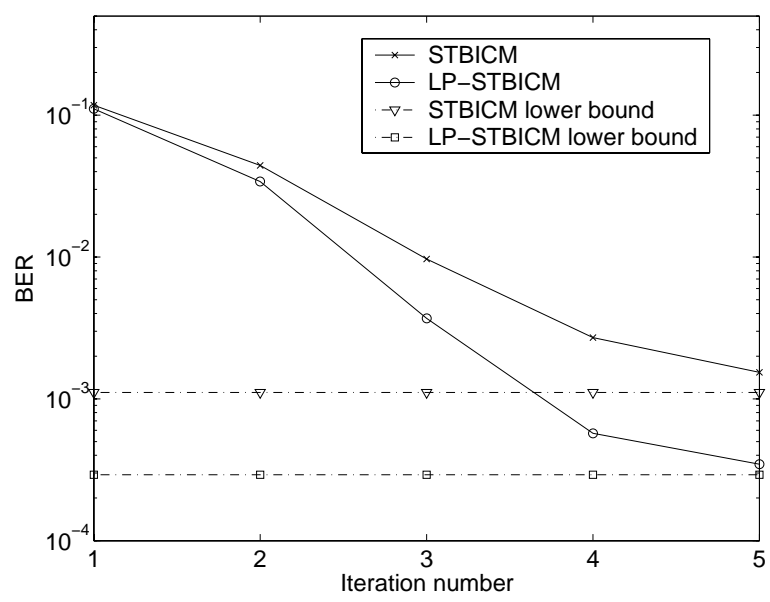

Fig. 3. Convergence Analysis at $\mathrm{SNR}=4 \mathrm{~dB}$

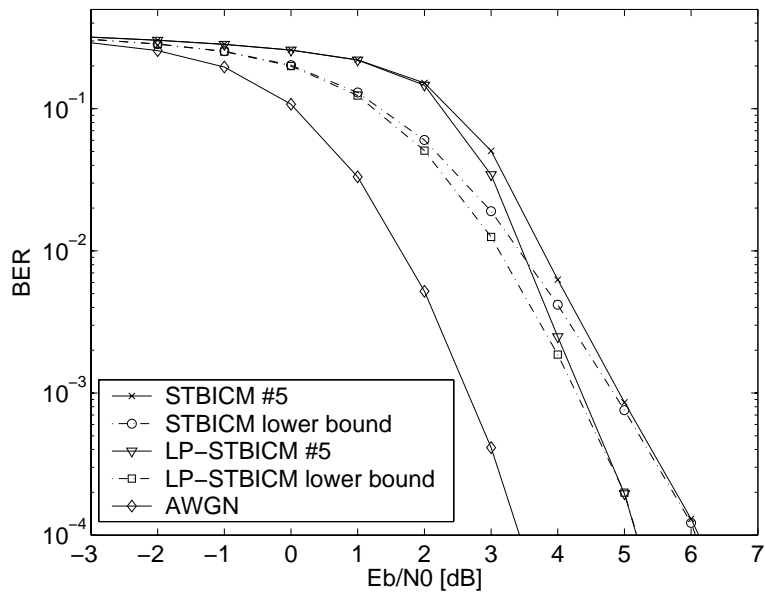

Fig. 4. Iterative receiver for $2 \times 2$ white Rayleigh flat fading channel

channel for which the CAI is greater. In the other hand, at high SNR, all the CAI is perfectly cancelled, $5^{\text {th }}$ iteration curves both tend to their respective lower bounds. If we compare LP-STBICM and BICM, we can notice that linear precoding bring an adding gain of $1 \mathrm{~dB}$ for $2 \times 2$ channel and $0.7 \mathrm{~dB}$ for $4 \times 4$ channel at a bit error rate of $10^{-4}$ owing to linear precoding which has the effect of increasing the global system diversity. As we can see on the LP-STBICM curves, slopes are near similar to the Gaussian one, thus Gaussian diversity is achieved.

\section{CONCLUSION}

The interest of linear precoding combined with orthogonal STBC has already been demonstrated. We show in this paper that the combination of linear precoding and spatial multiplexing is efficient as well. By iteratively removing the CAI, the PSIC equalizer restores the orthogonality essential for linear

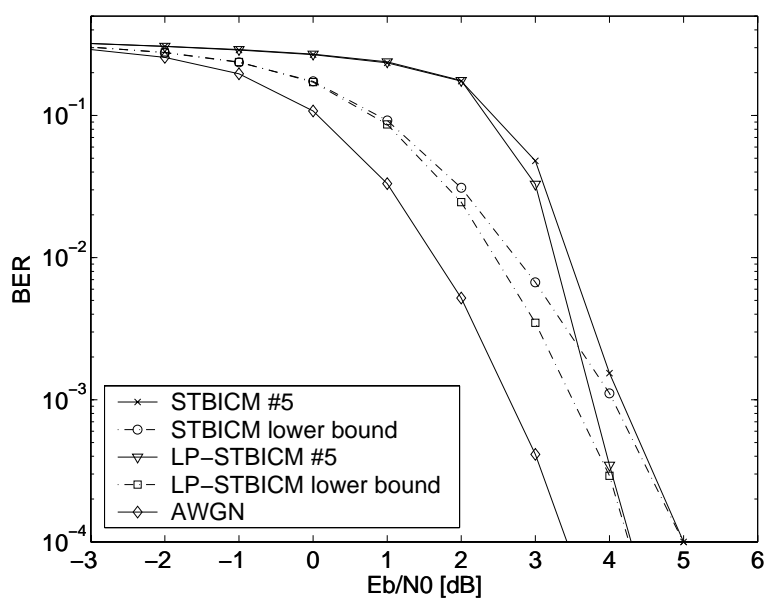

Fig. 5. Iterative receiver for $4 \times 4$ white Rayleigh flat fading channel

precoding. The proposed combination of linear precoding and STBICM makes possible the exploitation of MIMO capacity without sacrificing diversity and vice versa. Furthermore such diversity can be easily increased with the size of the precoding matrix at a linear cost of complexity. We demonstrate the efficiency of a sub-optimum receiver based on an iterative detection and decoding scheme. This receiver can be seen like a turbo-equalizer acting on the CAI instead of intersymbol interference. The main interest relies on the low complexity of each decoding stages. In fact this receiver can be easily applied to great antenna number systems providing very high date rate.

\section{REFERENCES}

[1] G. J. Foschini and M. J. Gans, "On limits of wireless communications in a fading environment when using multiple antenna," Wireless Pers. Commun., vol. 6, pp. 311-335, Mar. 1998.

[2] J. G. Foschini, "Layered space-time architecture for wireless communication in a fading environment when using multielement antennas," Bell Syst. Tech. Journal, vol. 1, pp. 41-59, Oct. 1996.

[3] M. Sellathurai and S. Haykin, "TURBO-BLAST for high-speed wireless communications," in Proceedings of $W C N C$ '00, Chicago, USA, Sept. 2000 .

[4] V. Tarokh, N. Seshadri, and R. Calderbank, "Space-time codes for high data rate wireless communication: performance criterion and code construction," IEEE Trans. Inform. Theory, vol. 44, no. 2, pp. 744-765, Mar. 1998.

[5] S. M. Alamouti, "A simple transmit diversity technique for wireless communications," IEEE J. Select. Areas Commun., vol. 16, no. 8, pp. 1451-1458, Oct. 1998.

[6] A. M. Tonello, "Space-time bit-interleaved coded modulation with an iterative decoding strategy," in Proceedings of VTC Fall'OO, Boston, USA, Sept. 2000, pp. 473-478.

[7] G. Caire, G. Taricco, and E. Biglieri, "Bit-interleaved coded modulation,” IEEE Trans. Inform. Theory, vol. 44, pp. 927-945, May 1998.

[8] J. J. Boutros and E. Viterbo, "Signal space diversity: a power and bandwith efficient diversity technique for the rayleigh fading channel," IEEE Trans. Inform. Theory, vol. 44, no. 4, pp. 1453-1467, July 1998. 
[9] A. Stamoulis, Z. Liu, and G. B. Giannakis, "Space-time block-coded OFDMA with linear precoding for multirate services," IEEE Trans. Signal Processing, vol. 50, no. 1, pp. 119-129, Jan. 2002.

[10] J. J. Boutros, N. Bresset, and L. Brunel, "Turbo coding and decoding for multiple antenna channels," in Proceedings of Proceedings of ISTC'03, Brest, France, Sept. 2003

[11] V. Le Nir and M. Hélard, "Reduced-complexity space-time block coding and decoding schemes with block linear precoding," IEE Elec. Letters, vol. 39 , no. 14 , pp. 1066-1068, July 2003.

[12] C. Douillard, A. Picart, P. Didier, M. Jézéquel, C. Berrou, and A. Glavieux, "Iterative correction of intersymbol interference: Turboequalization," Eur. Trans. Telecommunications, vol. 6, no. 5, Sept. 1995.

[13] A. Glavieux, C. Laot, and J. Labat, "Turbo equalization over a frequency selective channel,' in Proceedings of ISTC'97, Brest, France, Sept. 1997, pp. 96-102.

[14] C. Berrou, P. Adde, E. Angui, and S. Faudeil, "A low complexity softoutput viterbi decoder architecture," in Proceedings of ICC'93, Geneva, Switzerland, May 1993, pp. 737-740.

[15] M. Sellathurai and S. Haykin, "TURBO-BLAST for wireless communications: theory and experiments," IEEE Trans. Signal Processing, vol. 50, no. 10, pp. 2538-2546, Oct. 2002. 